\title{
The Behaviour of Metal Coated Carbon Fiber in Microwave Absorbing Composites
}

\author{
Y.X. Gan, C.Q. Chen and C.G. Li \\ Department of Materials Science and Engineering, \\ Beijing University of Aeronautics and Astronautics, \\ Beijing 100083, People's Republic of China
}

CONTENTS

Page

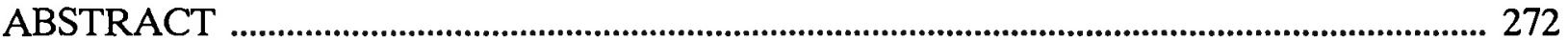

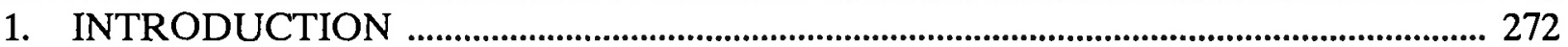

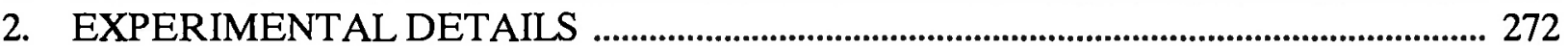

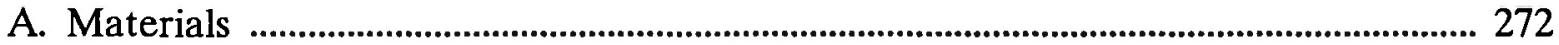

B. Preparation of the Specimen ................................................................................ 272

C. Measurements ............................................................................................................... 272

3. RESULTS AND DISCUSSION ............................................................................................... 273

A. Effect of the Metal Coated Fiber Addition on the Microwave Attenuation Property of the Composite ............................................................................................... 273

B. Effect of the Metal Coated Carbon Fiber Addition on the Electromagnetic Parameters of the Composite ......................................................................................... 273

C. Comparison of the Microwave Attenuation of the Calculated Model with the Experimental Result ................................................................................................ 274

D. The Resonant Effect of MCF …............................................................................ 275

E. The Near-Field Characteristic of the Metal Coated Carbon Fiber and the Analysis of the Microwave Absorbing Mechanism of the Composite Containing MCF ........................................................................................................... 276

F. The Microwave Attenuation Properties of the Types of Composites Containing MCF and Different Lossy Substances .......................................................... 278

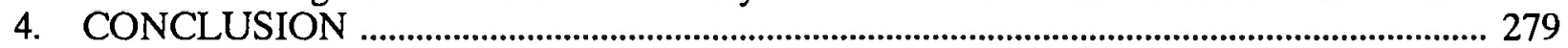

ACKNOWLEDGEMENTS ............................................................................................ 280

REFERENCES …................................................................................................................. 281 


\begin{abstract}
This research focuses on the behaviour of a metal coated carbon fiber (MCF) added to a microwave absorbing composite which has a multilayer structure. It was found that MCF can be used to adjust the electromagnetic parameters of the absorbing medium, to improve the attenuating property of the absorber and to widen the working frequency band width of the microwave. MCF works mainly in the mechanism of the resonant dissipation of incident microwave energy and can be applied to various absorbing mediums to fabricate composites. A discussion on the near-field characteristic of the MCF resonator based on the solution of Maxwell's equations is also presented.
\end{abstract}

\section{KEY WORDS}

metal coated carbon fiber, resonator, microwave absorbing composite

\section{INTRODUCTION}

Lightweight and thin broad band electromagnetic wave absorbing materials have long held researchers' interest $/ 1,2 /$. Besides ferrite, metal particles and macromolecules $/ 1,3-6 /$, a microwave absorbing composite material with electrical conductive fiber has been developed. Such a composite has the broad microwave attenuation frequency band due mainly to the resonant effect in the electromagnetic field followed by the loss of dielectric substances which changes incident electromagnetic energy into heat energy $2,7 /$.

Metallic wire, stainless steel fiber, aluminium coated polyester fiber, silver coated nylon fiber and metal coated carbon fiber can be used as a conductive resonator. In this paper, metal coated carbon fiber (MCF) was used. MCF is superior to others for the following advantages:

(1) MCF has a high specific strength ( $>2500 \mathrm{MPa}$ ) and a high specific modulus $\left(>2 \times 10^{5} \mathrm{MPa}\right)$. Thus, it can keep straight in the matrix. Furthermore, it is easier to sufficiently mix MCF with resin and lossy substances than other flexible fibers.

(2) The diameter of MCF is very fine, about $7 \mu \mathrm{m}$. In the high frequency range, the resonant loss is dependent on the added number of the conductive fiber and the total conductive area. So, the finer the fiber, the more effective the resonant loss.

(3) The specific weight of MCF is low. It is suitable for a thin, light and broad band microwave absorbing structure.

\section{EXPERIMENTAL DETAILS}

\section{A. Materials}

Metal coated carbon fibers (MCF) were prepared by a continuous electroplating process $/ 8 /$. Two kinds of metal coatings were used: copper coating and nickel coating. Both had a thickness of about 0.3 micron and were plated onto carbon fibers. The fibers were then cut into short fibers with a mean length of $3 \mathrm{~mm}$. Epoxy was used as an adhesive.

The electromagnetic lossy substances used were as follows: Mn-Zn ferrite, carbon black, clay, a sintered mixture of zirconia and magnesium oxide and pure epoxy resin.

\section{B. Preparation of the Specimen}

A calculated amount of MCF, lossy substance and resin adhesive were sufficiently mixed. The mixture was smeared on a $2 \mathrm{~mm}$ thick aluminium metal substrate layer by layer, until the thickness of the composite on the plate reached above $1.7 \mathrm{~mm}$. The composite was cured layer by layer at $120^{\circ} \mathrm{C}$ using infrared rays. The surface of the composite was polished on wet No. 400 emery paper. Fig. 1 shows the schematic drawing and cross sections of the material.

\section{Measurements}

The microwave attenuation property of the composite was tested on an HP8410C network analyser at the frequency range of $7 \sim 18.5 \mathrm{GHz}$. Measurement of permeability and permittivity were performed by the use of an HP8510B network analyser in the frequency range of $8.2 \sim 12.4 \mathrm{GHz}$. The aluminium substrate of the speci- 

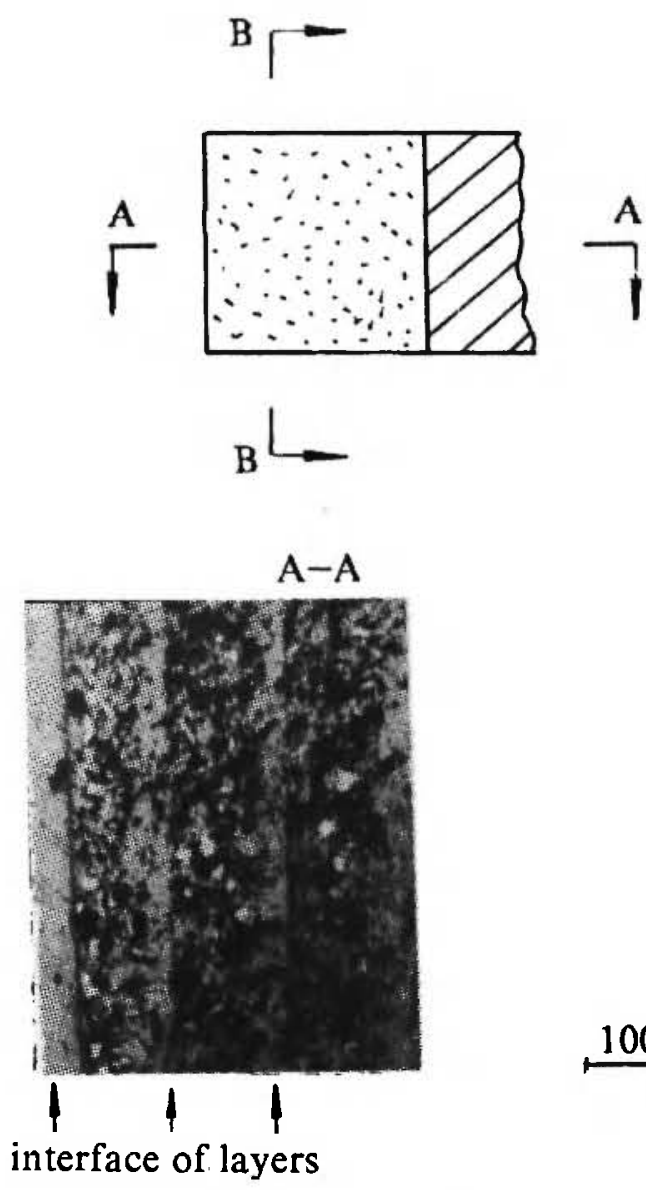

Fig. 1: Schematic drawing and cross section of the composite.

men was removed during permeability and permittivity measurements.

\section{RESULTS AND DISCUSSION}

\section{A. Effect of the Metal Coated Carbon Fiber Addition on the Microwave Attenuation Property of the Composite}

Fig. 2 compares the microwave attenuation property of the composite coating containing Ni coated carbon fibers with that of the composite coating containing no fibers. The two coating materials had the same matrix formula. The ratio of ferrite to epoxy was $25: 75$ in volume.

The curves show that the two materials almost have the same attenuation characteristic in the frequency ranges of $f<9.5$ and $f<13 \mathrm{GHz}$, whereas the former is superior to the latter in the frequency range of $9.5 \sim 13$ GHz. The composite with Ni coated carbon fibers generates a high peak of absorption with a considerable width. The width of the band with $-10 \mathrm{~dB}$ attenuation is $2.4 \mathrm{GHz}$ and $1.5 \mathrm{GHz}$ with $-15 \mathrm{~dB}$ attenuation. The maximum attenuation value is $-20 \mathrm{~dB}$.

\section{B. Effect of the Metal Coated Carbon Fiber Addition on the Electromagnetic Parameters of the Composite}

The permittivity and permeability of the two materials are listed in Table 1, which shows that the relative complex permeabilities of the two composites are nearly equal except for a slight declination in the real part of the complex permeabilities due to the Ni coated carbon fiber addition. As for permittivity, both the real and imaginary parts show a remarkable increase through the addition of $\mathrm{Ni}$ coated carbon fiber. 


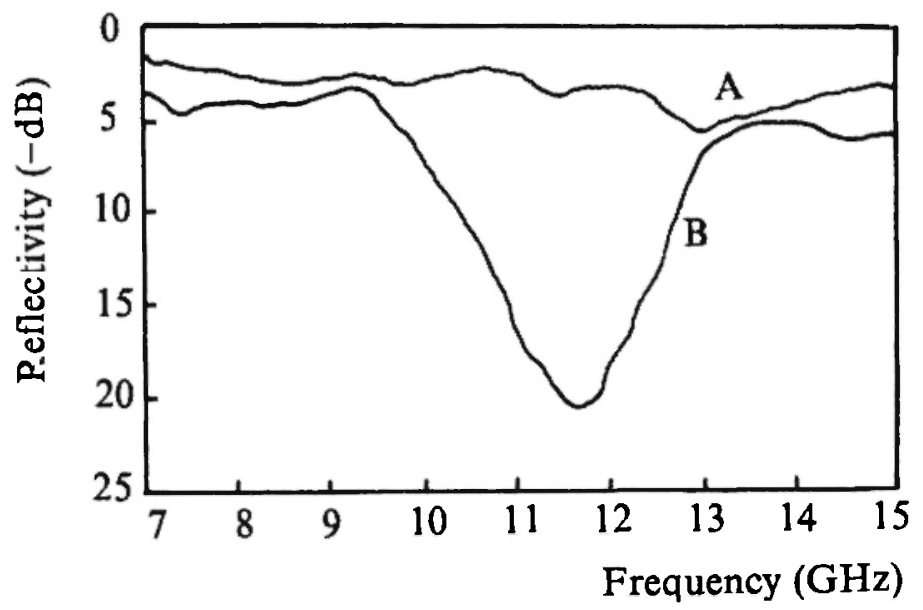

Fig. 2: Effect of the Ni coated carbon fiber addition on the microwave attenuation property of the composite coating containing ferrite.

A: with Ni coated carbon fibers

B: without Ni coated carbon fibers

TABLE 1

Permeability and Permittivity

\begin{tabular}{|c|c|c|c|c|c|c|c|c|}
\hline \multirow{2}{*}{$\begin{array}{c}\text { frequency } \\
\mathrm{f}(\mathrm{GHz})\end{array}$} & \multicolumn{4}{|c|}{ without $\mathrm{NiCF}$} & \multicolumn{4}{c|}{ with NiCF } \\
\cline { 2 - 9 } & $\mu_{\mathrm{r}}$ & $\mu_{\mathrm{i}}$ & $\varepsilon_{\mathrm{r}}$ & $\varepsilon_{\mathrm{i}}$ & $\mu_{\mathrm{r}}$ & $\mu_{\mathrm{i}}$ & $\varepsilon_{\mathrm{r}}$ & $\varepsilon_{\mathrm{i}}$ \\
\hline 8.2 & 1.09 & -0.04 & 3.86 & 0.07 & 1.04 & -0.04 & 22.71 & 1.76 \\
\hline 8.6 & 1.07 & -0.01 & 3.85 & 0.05 & 1.02 & -0.03 & 22.55 & 1.71 \\
\hline 9.0 & 1.09 & -0.03 & 3.85 & 0.08 & 1.03 & -0.02 & 22.65 & 2.36 \\
\hline 9.4 & 1.07 & -0.02 & 3.88 & 0.07 & 1.03 & -0.01 & 22.30 & 2.60 \\
\hline 9.8 & 1.09 & -0.03 & 3.85 & 0.10 & 1.08 & -0.00 & 21.94 & 2.54 \\
\hline 10.2 & 1.07 & -0.01 & 3.88 & 0.09 & 1.03 & 0.01 & 22.0 & 2.51 \\
\hline 10.6 & 1.07 & -0.01 & 3.88 & 0.09 & 1.03 & 0.01 & 22.13 & 2.69 \\
\hline 11.0 & 1.08 & -0.02 & 3.86 & 0.10 & 1.03 & 0.01 & 22.06 & 2.70 \\
\hline 11.4 & 1.08 & -0.02 & 3.89 & 0.08 & 1.04 & 0.00 & 22.60 & 2.76 \\
\hline 11.8 & 1.09 & -0.04 & 3.89 & 0.11 & 1.04 & 0.00 & 22.39 & 3.07 \\
\hline 12.2 & 1.09 & -0.03 & 3.89 & 0.09 & 1.04 & 0.00 & 22.63 & 3.14 \\
\hline 12.4 & 1.09 & -0.03 & 3.88 & 0.10 & 1.04 & 0.00 & 22.58 & 3.03 \\
\hline
\end{tabular}

C. Comparison of the Microwave Attenuation of the Calculated Model with the Experimental Result

Since NiCF is a short fiber and has a random orientation in the composite and its added amount is very limited (less than 0.3 per cent in volume content), we may consider the composite with $\mathrm{NiCF}$ as an approxi- mate homogeneous material. Thus, the electromagnetic wave transmission theory for a homogeneous monolayer coating on the metal substrate in the vertical incident can be applied to the calculation on the voltage reflectivity and power attenuation of the composite.

Let us assume that the electrical conductivity of the 
aluminium plate is infinite - that is, its inherent impedance is equal to zero. Then, we obtain the incident impedance of air and composite interface as follows:

$$
Z_{\text {ln }}=Z \cdot \tanh \left(\frac{2 \pi f}{c} \sqrt{\mu \varepsilon}\right)
$$

The voltage reflectivity, $\Gamma$, and the power attenuation value, $R$, can be expressed, respectively, as:

$$
\Gamma=\frac{Z_{\text {in }}-Z_{0}}{Z_{\text {in }}+Z_{0}}
$$

and

$$
R(d B)=20 l g|\Gamma|
$$

where $\mathrm{Z}$ is the characteristic impedance of the composite and

$$
Z=\sqrt{\mu_{0} / \varepsilon_{0}} \cdot \sqrt{\mu / \varepsilon}
$$

$\mathrm{Z}_{0}$ is the characteristic impedance of air and

$$
Z_{0}=\sqrt{\mu_{0} / \varepsilon_{0}} .
$$

$\mu_{0}$ and $\varepsilon_{0}$ are the permeability and permittivity of air; $\mu$ and $\varepsilon$ are the permeability and permittivity of the composite; $d$ is the thickness of the composite; $f$ is the microwave frequency; and $c$ is the speed of the microwave in the air.

Fig. 3 illustrates the calculated curve based on the data in Table 1. The thickness of the composite is 1.62 $\mathrm{mm}$. The microwave attenuation test result is also shown in the same figure for comparison. In the frequency range of $\mathrm{f}<9.5 \mathrm{GHz}$, no significant difference exists between the two curves. Nevertheless, the test curve has a high absorption peak with considerable width above $9.5 \mathrm{GHz}$. This means that the microwave absorption behaviour of the NiCF containing composite does not only depend on the electromagnetic parameters.

\section{The Resonant Effect of $M C F$}

To summarize, we may conclude that MCF cannot only improve the microwave attenuation property of absorbent by generating or elevating the absorption peak and broadening the absorption frequency band, but can also change or control the electromagnetic parameters mainly in the permittivity of the composite. This may be due to the resonant dissipation of MCF. MCF, as an electric dipoler, is in resonance with the incident wave which generates inductive current. The current then dissipates in the lossy matrix (epoxy and gasket). So the incident electromagnetic wave energy is changed into heat energy. In order to understand in detail the effect of the half wave length ( 0.47 wave length indeed) MCF

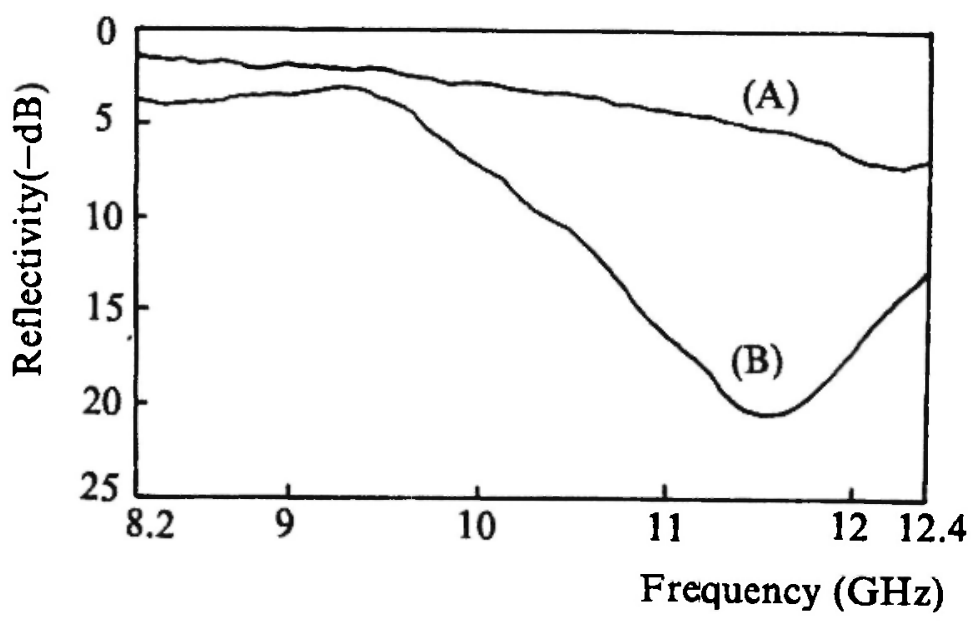

Fig. 3: Comparison of the microwave attenuation of the calculated curve with the experimental result.

(A) calculated curve

(B) experimental result 
resonator on the electromagnetic wave, the transmission, wave length of the electromagnetic wave in the composite containing MCF was calculated by

$$
\lambda=\frac{\lambda_{0}}{\sqrt{|\mu \varepsilon|}}
$$

where $\lambda_{0}$ is the wave length of the given frequency in the air; $\mu$ is the permeability of the composite; and $\varepsilon$ is the permittivity of the composite (the values of $\mu$ and $\varepsilon$ are listed in Table 1).

In the frequency range of $9.5 \sim 12.4 \mathrm{GHz}$, the calculated electrical wave length, $\lambda$, is about 7 to $9 \mathrm{~mm}$. And the central frequency of the attenuation peak in Fig. $3 b$ corresponds to an electrical wave length of $\lambda=7.3$ $\mathrm{mm}$. The added MCF has an average length of $3 \mathrm{~mm}$ which is approximately equal to the $0.47 \lambda$ of the electrical wave length corresponding to the central frequency.

The microwave attenuation test on another type of composite which contains $3 \mathrm{~mm}$ length $\mathrm{NiCF}$ and carbon black as the lossy substance also illustrates that the $\mathrm{NiCF}$ has the function of a half wave length resonator. Fig. 4 shows absorption peaks in the frequency range of 13 to $14 \mathrm{GHz}$. The measured permituivity and permeability of the composite are $\varepsilon=10.37-1.24 \mathrm{j}$ and $\mu=$ $0.97-0.04 \mathrm{j}$, respectively. The electrical wave length calculated using Eq. 4 is in the range of $6 \sim 7 \mathrm{~mm}$, which is double the length of NiCF in a good approximation.

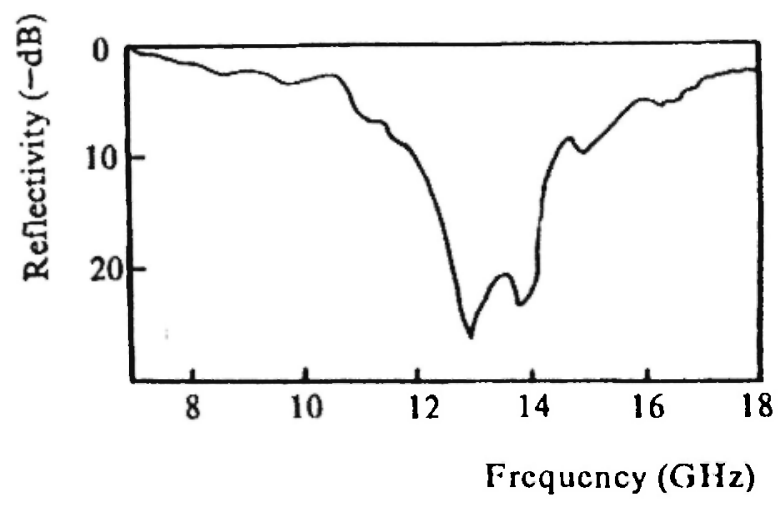

Fig. 4: Frequency scanning test result showing the half wavelength resonant effect of NiCF.
E. The Near-Field Characteristic of the Metal Coated Carbon Fiber and the Analysis of the Microwave Absorbing Mechanism of the Composite Containing MCF

No matter what the polarization pattern of the incident wave is, it exerts a stimulating voltage on the metal coated carbon fiber. An impressed current distribution of $J_{f}$ generates in the metal layer on the fiber, and $\mathrm{J}_{\mathrm{f}}$ results in the radiation of the $\mathrm{MCF}$ resonator. In order to determine the fields $E$ and $H$ (in fact, we are more interested in the $\mathrm{E}$ component in dealing with the composite containing the dielectric lossy matrix), we need to work with Maxwell's equations as given by Stutzman and Thiele and by Bi $/ 9,10 /$

$$
\begin{aligned}
& \nabla \times \mathbf{H}=\mathbf{J}_{\boldsymbol{f}}+j \omega \varepsilon \mathbf{E} \\
& \nabla \times \mathbf{E}=-j \omega \mu \mathbf{H} \\
& \nabla \cdot \mathbf{H}=0 \\
& \nabla \cdot \mathbf{E}=\frac{\rho,}{\varepsilon}
\end{aligned}
$$

Introducing the scalar and vector potential functions $\varphi$ and $A$, we get

$$
\begin{gathered}
\mathbf{H}=\frac{1}{\mu} \nabla \times \mathbf{\Lambda} \\
\mathbf{E}=-\nabla \varphi-\frac{\partial \boldsymbol{\Lambda}}{\partial t}
\end{gathered}
$$

Assuming that the electromagnetic field around the resonator is a time-harmonic field, we use Lorents condition of $\nabla \cdot \mathbf{A}=-\mu \varepsilon \frac{\partial \varphi}{\partial \mathrm{t}}$ and consider that the resonator resides in the lossy substance in order to obtain the D'Alembert equations presented in Eqs. 5 and 6, which are equivalent to Maxwell's equations presented in Eqs. 5-8:

$$
\begin{aligned}
& \nabla^{2} \mathbf{A}+\omega^{2} \mu \varepsilon \mathbf{A}=-\mu \mathbf{J}, \\
& \nabla^{2} \varphi+\omega^{2} \mu \varepsilon \varphi=-\frac{\mu_{f}}{\varepsilon}
\end{aligned}
$$

Consider volume $\tau$ ' bound by a closed surface. The solution for Eqs. 9 and 10 can be expressed as:

$$
A=\frac{\mu}{4 \pi} \int_{r} \frac{\mathbf{J}_{f} \cdot e^{-j k r}}{r} d t^{\prime}
$$




$$
\varphi=\frac{1}{4 \pi \varepsilon} \xi_{\tau_{t}} \frac{\rho_{t} \cdot \mathrm{e}^{-1 \mathrm{kr}}}{r} \mathrm{~d} \tau^{\prime}
$$

where $\rho_{f}$ is the impressed charge density and $k$ is equal to $\frac{\omega}{v}$ ( $v$ is the transmission speed of the electromagnetic field).

Using the near-field approximation $\mathrm{k} \ll \mathrm{l}$ in Eqs. 11 and 12 , yields

$$
\mathbf{A}=\frac{\mu}{4 \pi} \int_{,} \frac{I}{r} d \tau^{\prime}, \quad \varphi=\frac{1}{4 \pi \varepsilon} \int_{r} \frac{\rho_{f}}{r} d \tau^{\prime}
$$

A half wave length resonator is composed of multiple elements of current. As for an element of current of length $\Delta z$, we have

$$
\mathrm{J}_{f^{\prime}} d \mathrm{t}^{\prime}=e_{z} \mathrm{I} \Delta z
$$

The increment of the vector potential function generated by the element of current is

$$
\Delta \mathrm{A}=e \frac{\mu \mathrm{I} \Delta z}{4 \pi r}
$$

Then the electrical components of an arbitrary current element as illustrated in Fig. 5 may be written as:

$$
\begin{gathered}
\Delta \mathrm{E}_{\theta}=-\frac{j \mathrm{I} \sin \theta}{4 \pi \omega \varepsilon r^{3}} \Delta z \\
\Delta \mathrm{E}_{r}=-\frac{2 j \mathrm{I} \cos \theta}{4 \pi \omega \varepsilon r^{3}} \Delta z
\end{gathered}
$$

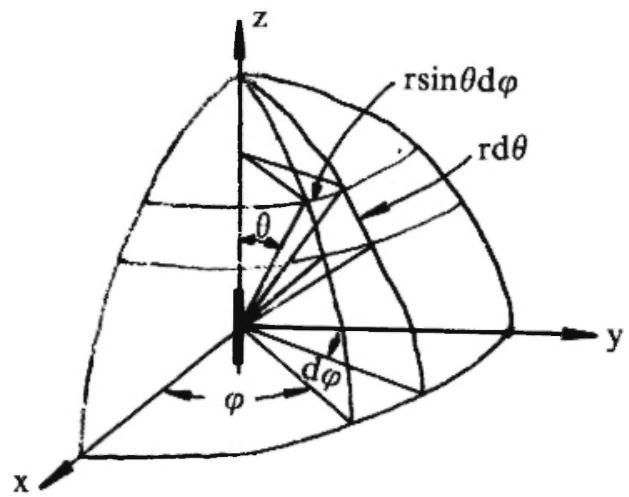

Fig. 5: Geometry used for field calculations of a metal coated carbon fiber resonator along the z-axis.
Dividing the resonator into infinite current elements and letting each element have an infinitesimal length, the electrical field becomes:

$$
\begin{aligned}
& \mathbf{E}_{\theta}=\int_{-1}^{\frac{1}{4}}\left(-\frac{j I \sin \theta}{4 \pi \varepsilon \omega r^{3}}\right) d z \\
& \mathbf{E}_{1}=\int_{-4}^{\frac{2}{4}}\left(-\frac{2 j \operatorname{las} \frac{D}{4 \pi \omega \varepsilon r^{2}}}{4}\right) d z
\end{aligned}
$$

Substituting $I=I_{m} \sin (2 \pi z / \lambda)$ into Eqs. 13 and 14 and using the near resonator condition $(z \approx r)$ gives

$$
\begin{aligned}
& \mathbf{E}_{\theta}=\int_{-\frac{\lambda}{4}}^{\frac{\lambda}{4}}\left(-\frac{j I_{m} \sin \theta \sin \frac{2 \pi z}{\lambda}}{4 \pi \varepsilon \omega r^{3}}\right) d z \\
& -\frac{-j I_{m} \sin \theta}{4 \pi \varepsilon(1)} \int_{-i}^{\frac{1}{4}} \frac{\sin \frac{2 \pi z}{2}}{z^{3}} d z \\
& \mathbf{E}_{r}=\int_{-\frac{\lambda}{4}}^{\frac{l}{4}}\left(-\frac{2 j I_{m} \cos \theta \sin \frac{2 \pi z}{2}}{4 \pi \varepsilon \omega r^{3}}\right) d z \\
& =\frac{-2 j I_{m} \cos \theta}{4 \pi c \omega} \int_{-\frac{4}{\lambda}}^{\frac{\lambda}{4}} \frac{\sin \frac{2 \pi z}{\lambda}}{z^{3}} d z
\end{aligned}
$$

where $I_{m}$ is the amplitude of the inductive current of the resonator. It can be seen from Eqs. 15 and 16 that $E_{\theta}$ and $E_{r}$ are time independent. That is, the electromagnetic field near the resonator is quasi-stationary. It is the quasi-stationary field that leads to a dissipative current (i) along the composed direction of $\mathrm{E}_{\theta}$ and $\mathrm{E}_{r}$, see Fig. 6.

From the experimental results given above, we know that the imaginary part of the permittivity of the composite $\left(\varepsilon_{\mathrm{i}}\right)$ is equal to 1.24. If we ignore the frequency hysterisis of the conductive ion in the composite, then

$$
\frac{\sigma}{\omega \varepsilon_{0}}-\varepsilon_{2}=1.24
$$

where $\sigma$ is the conductivity of the composite.

Since $\frac{1}{i \hat{v} \hat{v}}<\frac{\sigma}{\omega \varepsilon_{0}}<100$, the composite belongs to the lossy medium with a good loss ability to the electromagnetic wave energy. The dissipative current is absorbed in the composite by generating heat. 


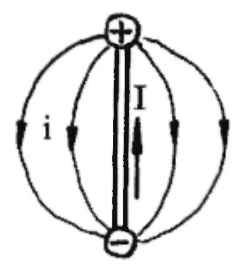

Fig. 6: Schematic showing the dissipative current distribution near a MCF resonator.

Therefore, MCF changes the electromagnetic field energy into dissipative current energy and the lossy substance absorbs the current energy by generating heat, which consists of the microwave absorbing processes of the composite material containing MCF resonators.

\section{F. The Microwave Attenuation Properties of Other} Types of Composites Containing MCF and Different Lossy Substances

For comparison, composites with MCF and different lossy substances including clay, ferrite, zirconic magnesium and carbon black were prepared, and composites without MCF were also made. They had the same volume content of epoxy. The thickness was $1.5 \mathrm{~mm}$. Figs. 7-11 show the microwave attenuation test results. It is clear that a good property in microwave absorption can be achieved by adding MCF. The width of the fre- quency band is $3.5 \mathrm{GHz}$ when reflectivity achie"es -10 $\mathrm{dB}$ and $2.0 \mathrm{GHz}$ with a $-15 \mathrm{~dB}$ attenuation. Maximum attenuation reaches $-35 \mathrm{~dB}$. Interestingly, pure epoxy with MCF generates considerable attenuation in the $K$ frequency band, though the microwave attenuation is not so remarkable when using only epoxy. It seems possible that the epoxy has a microwave absorption ability rather than being completely microwave permeable as reported in some of the literature. This also illustrates the improvement in the microwave absorption property by adding MCF.

The microwave attenuation property of composites changes with the addition of different lossy substances and different kinds of metal coatings on carbon fibers because different lossy substances have different inductive current dissipation abilities and the addition of different kinds of MCF results in different changes in the electromagnetic parameters of the composites. The work mechanism of the MCF as a half wave length resonator does not change.

Since MCF works in the resonating mechanism, the ability of absorbing the microwave of the composite containing MCF is not so dependent on the electromagnetic parameters and the thickness of the materials as is the Jaumann absorber and other types of electromagnetic wave absorbers. MCF is not only suitable for

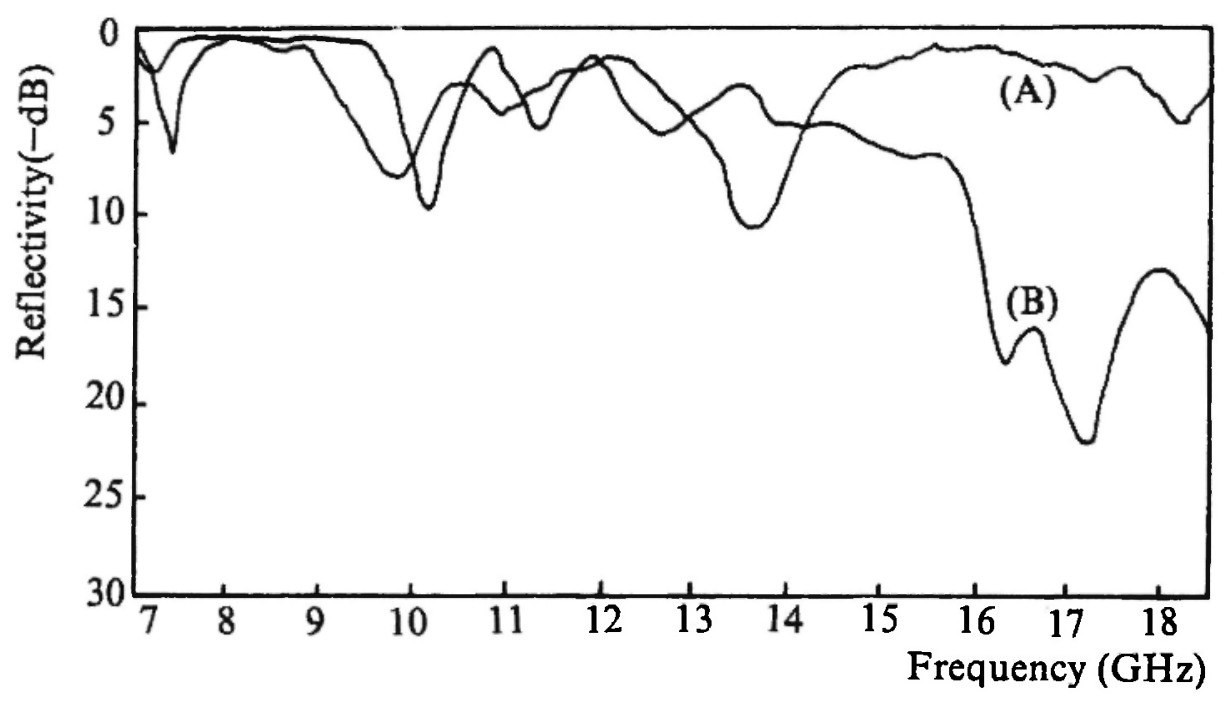

Fig. 7: Microwave attenuation property of pure epoxy and epoxy with MCF added.

(A) without Cu coated carbon fiber

(B) with Cu coated carbon fiber 


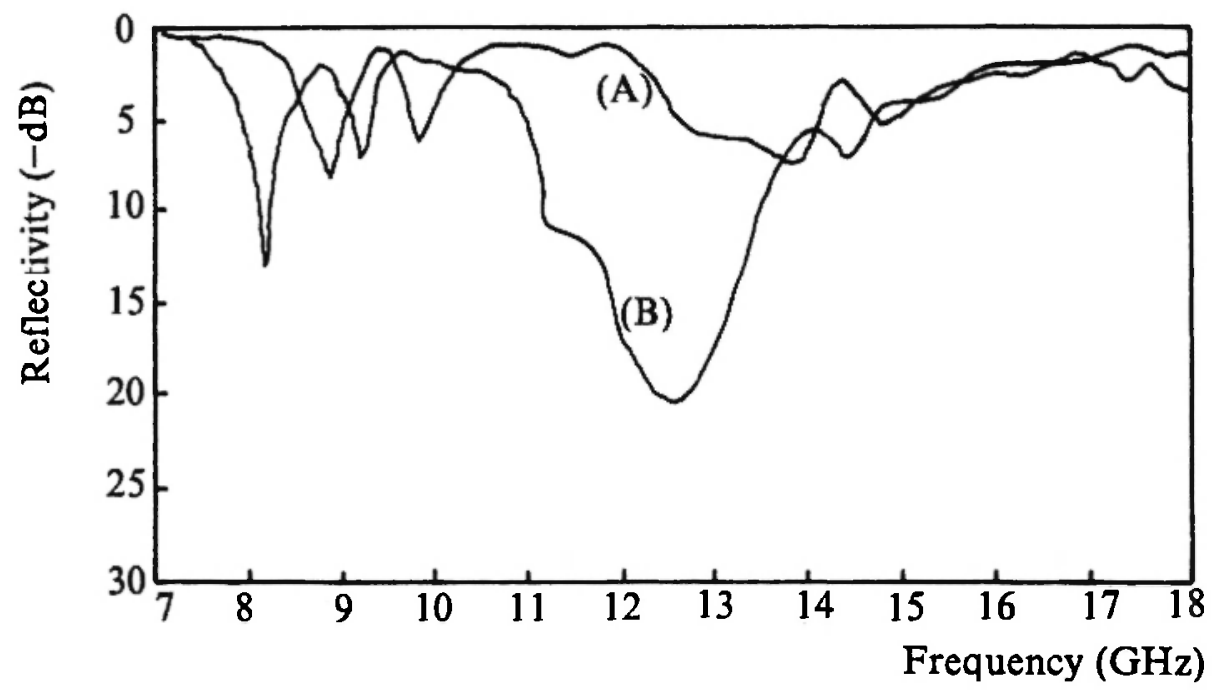

Fig. 8: Microwave attenuation property of the composites with zirconic magnesium.

(A) without $\mathrm{Ni}$ coated carbon fiber

(B) with $\mathrm{Ni}$ coated carbon fiber

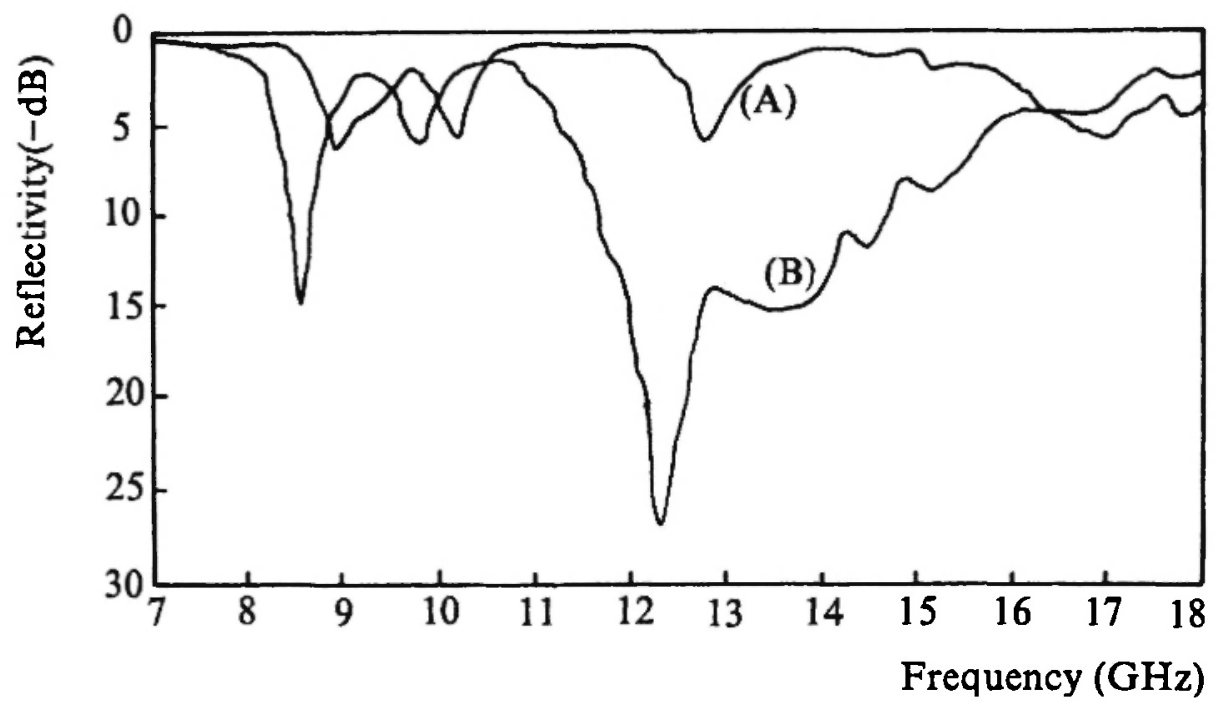

Fig. 9: Microwave attenuation propeı,, of the composite containing clay. (A) without $\mathrm{Ni}$ coated carbon fiber

(B) with $\mathrm{Ni}$ coated carbon fiber

a magnetic lossy matrix, but also for a dielectric medium, which is shown in Figs. 7-11. Furthermore, designability of composites in microwave radiation absorbing can be by adjusting the content, length of the fiber and the kind of coating on the fiber. Therefore, MCF may receive more and more notice in the development of multilayer, thin, light, broad band electromagnetic wave absorbers.

\section{CONCLUSION}

1. MCF has the advantages of high strength, high stiffness, light weight, fine in diameter and a good electrical property in ultrahigh frequency electromagnetic field. It is a superior additive for microwave attenuation composite materials.

2. MCF can change the electromagnetic parameters of 


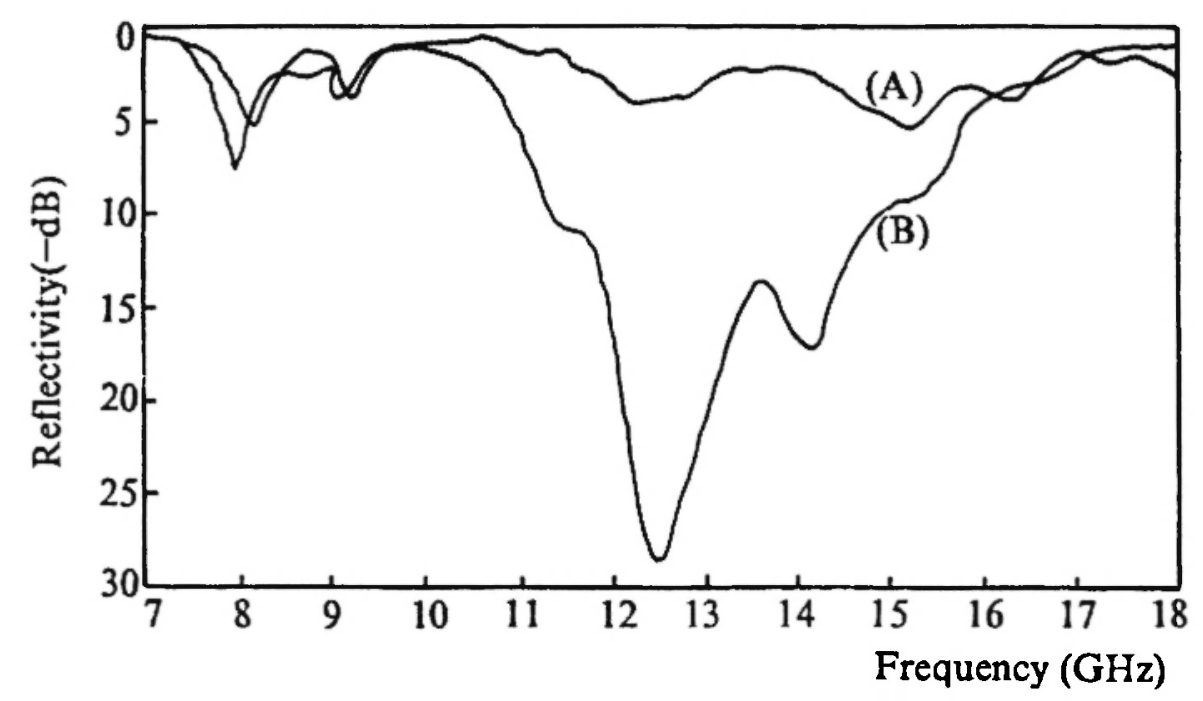

Fig. 10: Microwave attenuation property of the composite containing ferrite.

(A) without $\mathrm{Ni}$ coated carbon fiber

(B) with $\mathrm{Ni}$ coated carbon fiber

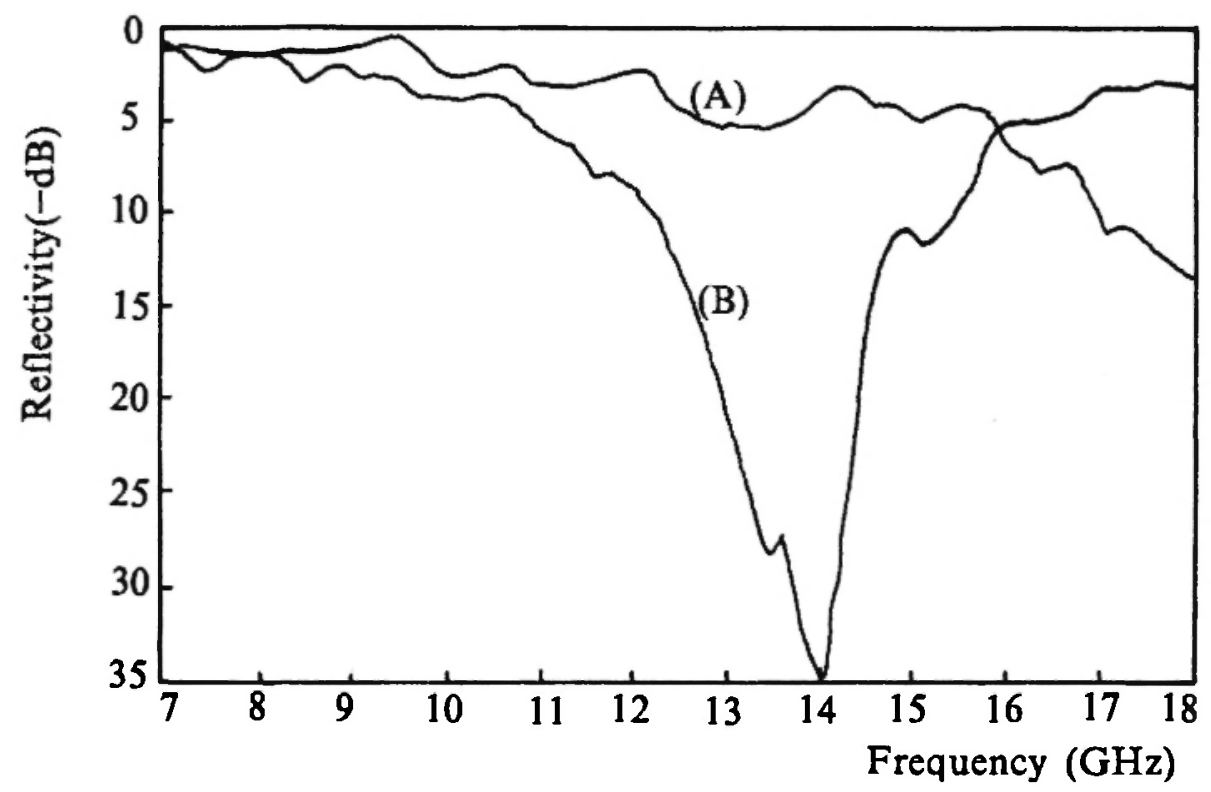

Fig. 11: Microwave attenuation property of the composite containing carbon black.

(A) without $\mathrm{Ni}$ coated carbon fiber

(B) with Ni coated carbon fiber

the absorber, mainly in a complex dielectric constant.

3. MCF, as a resonator, works with lossy substances to change incident microwave energy to heat energy.

4. The improvement on the microwave attenuation property by adding MCF is not only in the magnetic lossy materials but also in the dielectric absorbents.

\section{ACKNOWLEDGEMENT}

The authors would like to acknowledge the support 
of the National Nature Science Foundation of China under Grant Numbers 59103023 and B-587004. Thanks are also due to Mr. P. Yih for his assistance in preparing the composites.

\section{REFERENCES}

1. WALLING, E.W., Gunnar Lindquist Camouflage Material United States Patent 4,001,827 Jan. 4, 1977.

2. STONIER, R.A., SAMPE Journal, 28 (5), 9-18 (1991).

3. YAMASHITA, H. et al., Radio Wave Absorptive Gasket United States Patent 4,731,286 Mar. 15, 1988.

4. ISHINO, K. et al., Microwave Absorber United
States Patent 4,003,840 Jan. 18, 1977.

5. DAWSON, M.H. et al., Anti-Radar Meains and Techniques United States Patent 4,173,018 Oct. 30, 1979.

6. WAN, X.M., Astronautical Materials Techniques, 4-5, 28-32 (1984).

7. XIU, HJ., Astronautical Materials Techniques, 2 , 57-59 (1987).

8. YIH, P., CHEN, B.K. and WU, Y.S., SAE Technical Paper Series 850711, 21st Annual Airline Plating and Metal Finishing Forum Atlanta, Georgia, Feb. 18-21, 1985.

9. STUTZMAN, W.L. and THIELE, G.A., Antenna Theory and Design, John Wiley and Sons Inc., 1991, pp6.

10. BI, D.X., The Theory of Electromagnetic Field, Electronic Industry Press of China, 336 (1985). 
